\title{
Resistive and viscous dynamics of finite-amplitude shear waves at a magnetic $X$-point
}

\author{
I. J. D. Craig ${ }^{a)}$ \\ Department of Mathematics, University of Waikato, Private Bag 3105, Hamilton, New Zealand \\ Yuri E. Litvinenko ${ }^{\text {b) }}$ \\ Institute for the Study of Earth, Oceans, and Space, University of New Hampshire, Durham, \\ New Hampshire 03824-3525
}

(Received 11 July 2005; accepted 11 October 2005; published online 22 November 2005)

\begin{abstract}
The dynamics and dissipation of axial shear waves, superposed on a planar magnetic $X$-point in a resistive viscous incompressible plasma, are analyzed numerically and analytically. The interplay of viscous and resistive effects is demonstrated by deriving solutions for various values of the scalar coefficients of viscosity and resistivity. These solutions show that viscous-resistive coupling can dramatically affect the global energy dissipation. When either viscosity or resistivity vanishes, the solutions are characterized by oscillatory decaying eigenmodes that maintain equipartition between the magnetic and kinetic energies. This behavior persists when resistivity is the dominant dissipation mechanism. When viscosity is the dominant dissipation mechanism, initial oscillations are followed by exponential decay at sufficiently long times. The applicability of the results to flares in solar active regions, where the viscous Reynolds number can be much smaller than the resistive one, is discussed. (C) 2005 American Institute of Physics. [DOI: 10.1063/1.2132249]
\end{abstract}

\section{INTRODUCTION}

Theoretical studies of magnetic merging and reconnection typically ignore plasma viscosity. This is usually justified by the fact that "topological" magnetic energy associated with nonpotential magnetic fields, say, in the solar corona, can only be released by resistive effects. ${ }^{1}$ Thus electric current singularities, formed in an ideal plasma, cannot be resolved by viscosity alone, or indeed by any mechanism that neglects resistive dissipation.

Viscous effects, however, can strongly influence the magnetic field dynamics and dissipation in several important ways. Viscosity is known to limit reconnection exhaust speeds $^{2}$ and to significantly modify the geometry of flux pile-up reconnection. ${ }^{3}$ Viscous heating can be comparable to or even greater than Ohmic heating at sites of magnetic merging, where the plasma speed has large gradients. ${ }^{4}$ More generally, the viscous Reynolds number can be many orders of magnitude smaller than the magnetic Reynolds number for plasmas of solar active regions. Therefore viscosity should play an important role in a wide range of active phenomena in the solar corona. ${ }^{5}$

Previous studies of viscoresistive dynamics focussed on magnetic reconnection in the plane of a two-dimensional magnetic $X$-point. ${ }^{2,6-9}$ It has been established that a dissipation length scale emerges when viscosity and resistivity act together, which determines the rate of energy dissipation. The goal of this paper is to complement the previous studies by investigating the roles of viscosity and resistivity in the dynamics of finite-amplitude axial field disturbances in a potential magnetic $X$-point geometry. This problem allows a simple description of viscous and resistive damping and

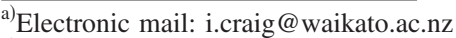

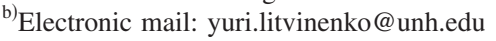

should provide a useful guide for wave dissipation in more general magnetic topologies. The resistively damped solution has been given earlier. ${ }^{10}$ Now we explore the viscoresistive coupling by studying numerical and analytical solutions that describe steady and transient shear wave disturbances. Although the bulk of the paper deals with time-dependent damping based on an isotropic viscosity, applicable for relatively weak magnetic fields, we also use steady-state solutions to investigate the likely impact of anisotropic viscosity.

The paper is organized as follows. In Sec. II we introduce the magnetohydrodynamic (MHD) equations that govern the evolution of the planar and axial components of the velocity and magnetic field near a two-dimensional magnetic $X$-point. Numerical solutions are presented, which show the viscoresistive decay of finite-amplitude axial field disturbances. A detailed theoretical interpretation of the numerical results is presented in Sec. III, where exact analytical solutions are derived in several cases. Steady-state solutions are presented in Sec. IV, which are used to discuss the relevance of models based on isotropic viscous stress tensor to the active phenomena in the solar corona. We summarize our findings in Sec. V.

\section{TRANSIENT SHEAR WAVES AT MAGNETIC $X$-POINTS}

We consider the magnetic $X$-point geometry shown in Fig. 1. The background potential field is perturbed by shear wave disturbances, polarized perpendicular to the plane of the $X$-point, which vanish at $x= \pm 1$. Our purpose is to examine the dynamics of such disturbances for arbitrary levels of viscous and resistive dissipation. 


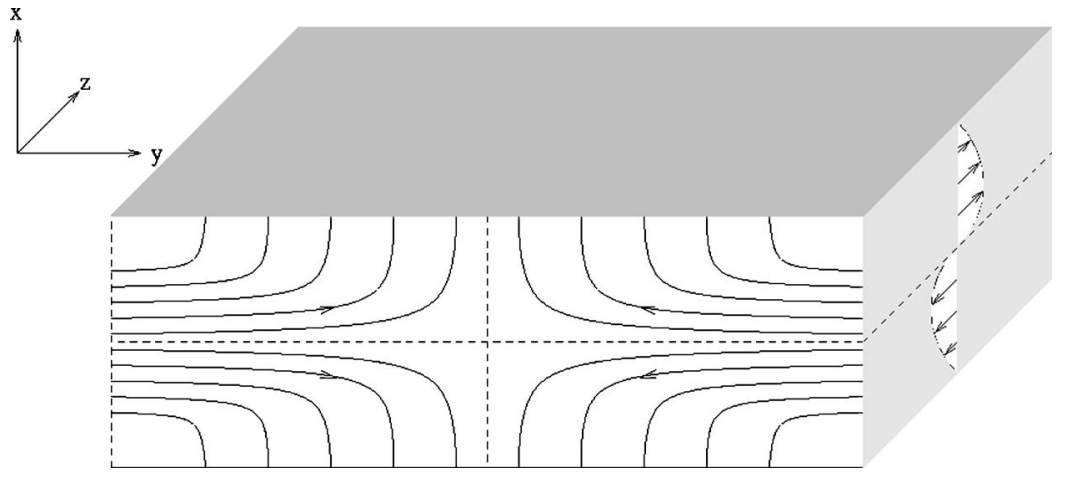

FIG. 1. Geometry of shear wave disturbances about an equilibrium $X$-point. The shearing is perpendicular to the plane of the $X$-point.

\section{A. Governing MHD equations}

The incompressible MHD equations in "two and a half dimensions" provide the basis for our analysis. We adopt a dimensionless formulation in which lengths, magnetic field intensities, and plasma density are normalized by reference values. Velocities and times are then measured in units of the Alfvén speed and time. As far as the dissipation coefficients are concerned, the dimensionless resistivity $\eta$ is the inverse Lundquist number, and the dimensionless viscosity $\nu$ is the inverse Reynolds number, based on the Alfvén speed. Both coefficients are much less than one in many astrophysical problems.

We use the stream function representation for the plasma velocity,

$$
\mathbf{v}(x, y, t)=\nabla \phi \times \hat{\mathbf{z}}+W \hat{\mathbf{z}},
$$

and the flux function representation for the magnetic field,

$$
\mathbf{B}(x, y, t)=\nabla \psi \times \hat{\mathbf{z}}+Z \hat{\mathbf{z}},
$$

which identically satisfy the divergence-free conditions for $\mathbf{v}$ and $\mathbf{B}$. Using the Poisson bracket $[\psi, \phi]=\psi_{x} \phi_{y}-\psi_{y} \phi_{x}$, where $\psi_{x}$ denotes $\partial \psi / \partial x$, etc., the nondimensionalized momentum and induction equations can be written as follows:

$$
\begin{aligned}
& \left(\nabla^{2} \phi\right)_{t}+\left[\nabla^{2} \phi, \phi\right]=\left[\nabla^{2} \psi, \psi\right]+\nu \nabla^{4} \phi, \\
& \psi_{t}+[\psi, \phi]=\eta \nabla^{2} \psi, \\
& W_{t}+[W, \phi]=[Z, \psi]+\nu \nabla^{2} W, \\
& Z_{t}+[Z, \phi]=[W, \psi]+\eta \nabla^{2} Z .
\end{aligned}
$$

For collisional coronal plasmas involving weak magnetic fields, we have the typical magnitudes $\nu \simeq 10^{-4.5}$ and $\eta$ $\simeq 10^{-14.5}$ (see Sec. IV). Below we explore the evolution of the axial fields $Z$ and $W$ in the presence of small but arbitrary $\nu$ and $\eta$. Note that we have adopted simple Laplacian forms for the viscous and resistive dissipation terms. In the presence of a sufficiently strong magnetic field, however, the plasma viscosity becomes highly anisotropic and requires a tensor description, as discussed in Sec. IV.

\section{B. Shear-wave axial modes}

It is well known that models with the potential background field, $\psi=x y$, permit a wide class of exact analytical solutions, both time-dependent and steady-state. ${ }^{11-13}$ If we restrict attention to disturbances polarized perpendicular to the plane of the $X$-point, the simplest solutions are based on the one-dimensional forms $W=W(x, t)$ and $Z=Z(x, t)$. Planar equations are satisfied by taking $\psi=x y$ and $\phi=0$, in which case Eqs. (5) and (6) reduce to

$$
\begin{aligned}
& W_{t}=x Z_{x}+\nu W_{x x}, \\
& Z_{t}=x W_{x}+\eta Z_{x x} .
\end{aligned}
$$

Despite the simplicity of this system, it does not seem possible to obtain global analytical solutions, valid for arbitrary $\nu$ and $\eta$. Our strategy is therefore to perform numerical simulations in the bounded region $|x| \leqslant 1$ and to augment the simulations with exact solutions in certain special cases. For numerical purposes we adopt boundary conditions appropriate to the slab geometry illustrated in Fig. 1, namely

$$
W(0)=W( \pm 1)=0, \quad Z(0)=Z_{x}( \pm 1)=0 .
$$

These conditions, based on the idealization of line-tying on the upper and lower boundaries, effectively break the symmetry between the magnetic and velocity fields, a point to which we return below.

We emphasize from the outset that the behavior of the system can change significantly when both $\eta$ and $\nu$ are nonvanishing. When viscosity and resistivity act together to dissipate the magnetic and kinetic energies, the solution can possess a viscoresistive length scale,

$$
x_{s}=(\eta \nu)^{1 / 4} .
$$

This scale can be deduced dimensionally, by balancing the terms on the right-hand sides of Eqs. (7) and (8). The emergence of this length scale in viscous merging problems in other geometries has been noted in the past, ${ }^{2,7-9}$ but its role in controlling shear wave dissipation has not been explored.

\section{Viscous and resistive losses}

Consider first the wave properties of the ideal system, described by Eqs. (7) and (8) in the limit $\eta=\nu=0$. This sys- 


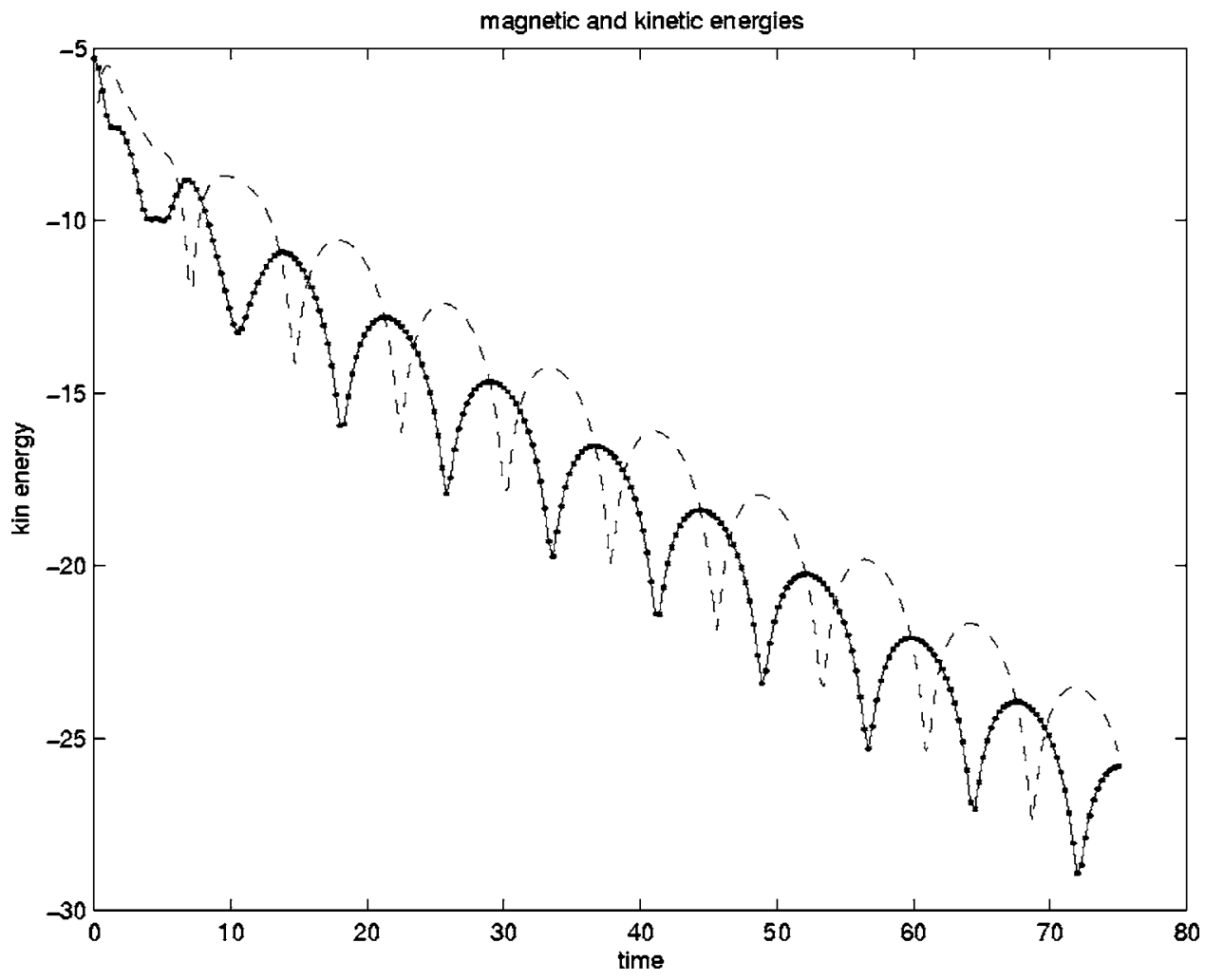

FIG. 2. Oscillatory decay of magnetic (dashed) and kinetic (solid) energies for $\eta=10^{-2.5}, \nu=10^{-3.5}$.

tem is simplified by working with the Elsasser variables $Z \pm W$, which are governed by the characteristics $\xi^{ \pm}=t \pm \ln x$. Ideal modes take the form

$$
\begin{aligned}
& Z=g(t+\ln x) \pm h(t-\ln x), \\
& W=g(t+\ln x) \mp h(t-\ln x),
\end{aligned}
$$

and conservation of wave energy is reflected by the invariance of the wave envelopes $g\left(\xi^{+}\right)$and $h\left(\xi^{-}\right)$.

Of particular interest is the continual steepening of the inward propagating wave $g\left(\xi^{+}\right)$as it approaches $x=0$. If $h$ vanishes initially, the system will maintain equipartition with $Z=W=g$ and $h=0$ as the wave travels inward. The buildup of steep gradients, $Z_{x}=W_{x}=g^{\prime}\left(\xi^{+}\right) / x$, can be arrested only by resistive and viscous effects. As shown in the Appendix, in the case of a freely propagating shear wave (11), viscosity (resistivity) has the effect of preferentially damping the kinetic (magnetic) energy of the disturbance.

In general, the energy loss rate due to viscous and resistive dissipation is given by

$$
\mathcal{E}_{t}=-\int\left[\nu\left(W_{x}\right)^{2}+\eta\left(Z_{x}\right)^{2}\right] \mathrm{d} V .
$$

Such losses, however, cannot be identified with the decay of the spatially integrated energy density $\frac{1}{2}\left(W^{2}+Z^{2}\right) .{ }^{10}$ This is because there can be a Poynting flux through the channel, which leaks energy from any fixed volume. Energy transfer by Poynting flux will occur at an Alfvénic rate, which may be unrelated to the volumetric heating rate. This point is crucial to the interpretation of the global wave energy loss in the system.

\section{Numerical examples of shear wave decay}

Figures 2-4 show typical computed solutions of Eqs. (7) and (8), based on the initial conditions $W(x, 0)=\sin (\pi x)$, $Z(x, 0)=0$. Wave energy dissipation is represented by time plots of the global magnetic and kinetic energies. Note that our choice of initial conditions guarantees odd solutions for both $W$ and $Z$, so it is sufficient to consider the solutions in the region $0 \leqslant x \leqslant 1$.

Figure 2 shows the case where resistivity dominates, $\eta$ $>\nu$. We observe that there is a continual oscillatory interchange of magnetic and kinetic energies as the global energy declines exponentially. By contrast, Fig. 3 shows the case where viscosity dominates, $\eta<\nu$. Now the initial oscillatory phase eventually gives way to a monotonic exponential decay in which the kinetic and magnetic energies are well separated. The dominance of viscosity is reflected in the stronger dissipation of the kinetic energy component of the fluid.

Finally, Fig. 4 shows the ratio of the kinetic to magnetic energies in the limiting case $\nu \gg \eta$. Oscillatory eigenmodes are still present but there is a clear separation in the global energies, consistent with the $t^{-2}$ power law. We argue below that a self-similar mode emerges, which gives rise to the power-law separation of the energies.

\section{ANALYSIS OF VISCOUS AND RESISTIVE DAMPING}

To interpret the numerical results of Sec. II, we need to investigate how shear waves evolve in the presence of both resistivity and viscosity. We first discuss the case of a single dissipation mechanism and then proceed to analyze the general case of nonvanishing $\eta$ and $\nu$. 


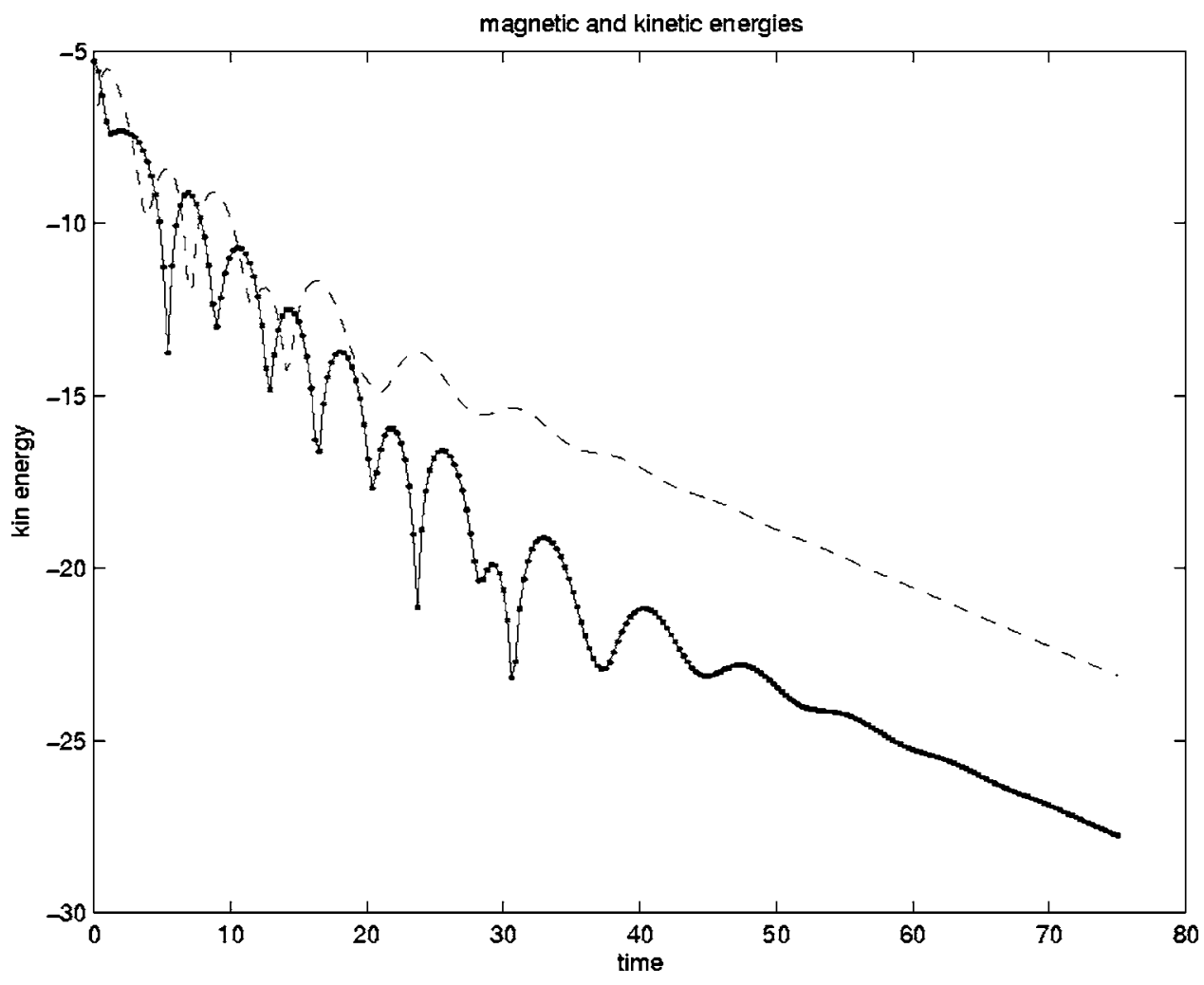

FIG. 3. Magnetic (dashed) and kinetic (solid) energy decay for $\eta=10^{-3.5}, \nu$ $=10^{-2.5}$. In contrast to the oscillatory profile of Fig. 2, the decay is monotonic for sufficiently long times.

\section{A. Viscously or resistively damped eigenmodes}

Motivated by the oscillatory character of Figs. 2 and 3, we begin by seeking eigenfunction solutions to Eqs. (7) and (8), subject to boundary conditions (9). Consider first the case of vanishing resistivity.

When $\eta=0$ we can eliminate $Z(x, t)$ and obtain a wave equation for $W(x, t)$, namely

$$
W_{t t}=x\left(x W_{x}\right)_{x}+\nu W_{x x t} .
$$

This equation can be solved by assuming that $W(x, t)$ $=e^{\lambda t} W(x)$, where $W(x)$ is a complex eigenfunction associated with an eigenvalue $\lambda=\alpha+i \omega$. It follows that

$$
\lambda^{2} W=x\left(x W^{\prime}\right)^{\prime}+\nu \lambda W^{\prime \prime},
$$

and a solution of the form

$$
W(s)=\sinh (\lambda s), \quad s=\ln \left(\frac{x}{\sqrt{\nu \lambda}}+\sqrt{1+\frac{x^{2}}{\nu \lambda}}\right)
$$

satisfies the inner boundary condition $W(0)=0$. The requirement that $W$ vanishes on the outer boundary $s \simeq|\ln (2 / \sqrt{\nu \lambda})|$ yields the dispersion relation

$$
\lambda \ln \frac{2}{\sqrt{\nu \lambda}}=(n+1) i \pi
$$

This equation defines a sequence of discrete eigenvalues $\lambda_{n}$, and $n=0$ determines the fundamental mode.

If the viscous coefficient $\nu$ is small enough, we expect oscillatory standing-wave solutions that decay relatively weakly. In this case $|\alpha / \omega| \ll 1$, and it is a simple matter to deduce the oscillation frequency and damping rate of the mode. We find that

$$
\omega_{n} \simeq \frac{2 \pi}{\ln (4 / \nu)}(n+1), \quad \alpha_{n}=-\frac{\omega_{n}^{2}}{2(n+1)} .
$$

These rates are fast in the sense that they depend only on $\ln \nu$, so the waves decay rapidly even when viscosity is small. As anticipated in Sec. II C, however, we can attribute the decay to the Poynting flux along the channel rather than to the local energy dissipation. In fact our analysis shows that the fundamental $n=0$ eigenmode is associated with the diffusion length scale of order $\nu^{1 / 2}$. Hence the rate of viscous energy dissipation is of order

$$
\mathcal{E}_{t} \simeq W_{0}^{2} \nu^{1 / 2},
$$

where $W_{0}$ is the velocity amplitude. Clearly the rate of wave decay and the actual rate of energy dissipation can differ dramatically because of the loss of energy from the system.

An analogous result, leading to the dissipation rate that scales as $\eta^{1 / 2}$, is obtained when $\nu=0$ but the electric resistivity $\eta \neq 0$. In this case $Z(x)$ is the primary dependent variable and the outer boundary condition is met by taking $Z_{x}(1)=0 .{ }^{10}$ We conclude that when either viscosity or resistivity acts alone, there is a well-defined oscillatory decay in which the magnetic and velocity fields are tied together by the constraint of energy equipartition.

These results suggest that the breakdown of energy equipartition requires the combined influence of viscosity and resistivity. Although this interpretation is substantially correct, we should bear in mind that we are dealing with a complex, non-self-adjoint system in which the completeness of the eigenmodes cannot be guaranteed. For example, as Fig. 4 indicates, self-similar modes can develop at large times, which preferentially damp the global kinetic energy in 


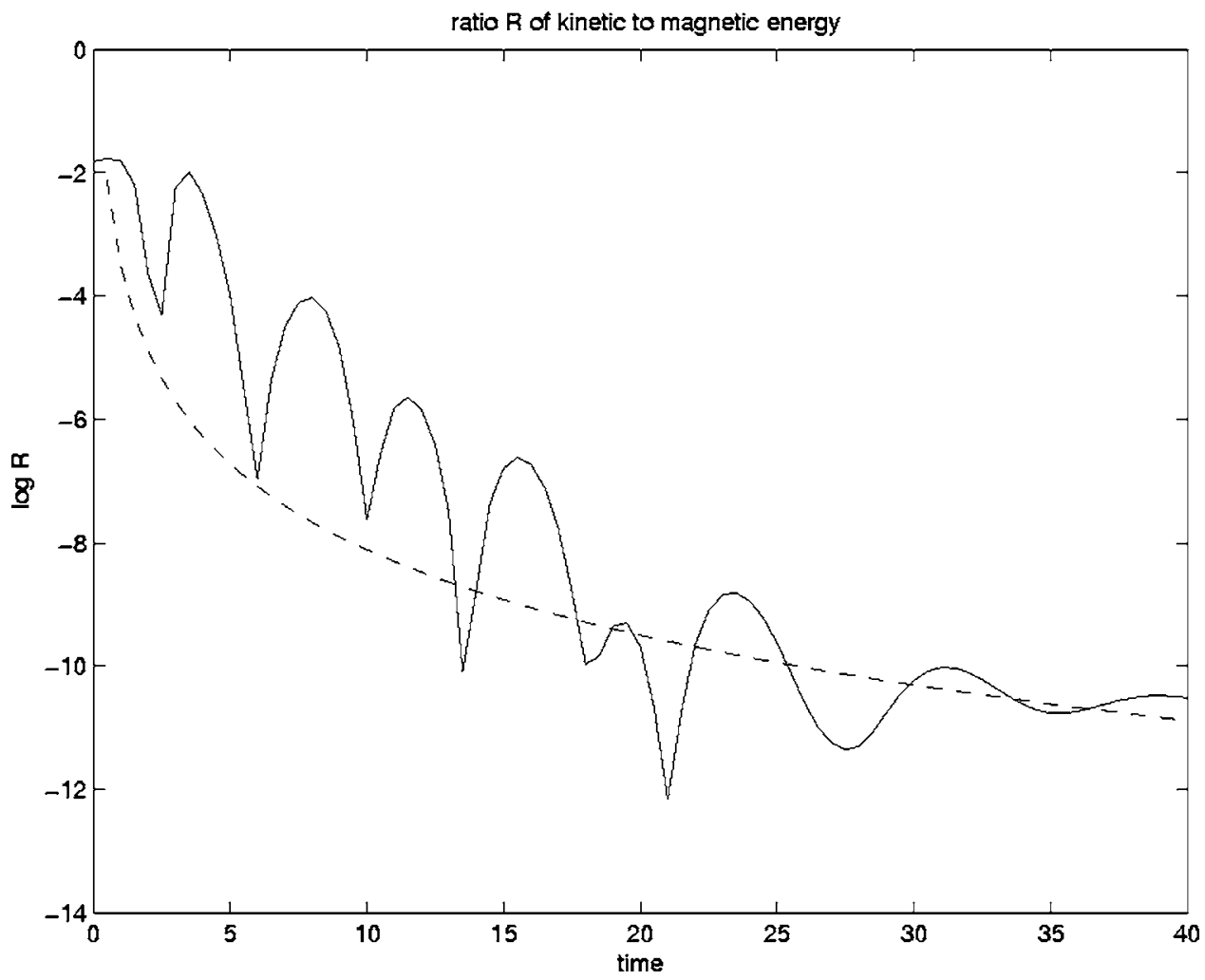

FIG. 4. Ratio of kinetic to magnetic energies for the case $\eta=10^{-8}, \nu$ $=10^{-2.5}$. The oscillatory eigenmode behavior is still evident but there is now a separation of energies with time, consistent with the power law $\sim t^{-2}$ of the monotonic self-similar mode.

the case $1 \gg \nu \gg \eta>0$. Such modes, while playing only a minor role in the overall energetics, appear to be a recurrent feature of the late decay for $\nu \gg \eta$.

\section{B. The self-similar mode}

To understand how self-similar behavior might arise, we note that, in the limiting case $\eta \rightarrow 0$, Eqs. (7) and (8) admit an approximate solution based on the variable $\zeta=x^{2} t / \nu$. The scale $\zeta<1$ determines a viscous boundary layer for the solution. Specifically, if $W$ varies slowly enough with time, Eqs. (7) and (8) lead to

$$
\nu \frac{\partial}{\partial x}\left(\frac{Z_{t}}{x}\right)+x Z_{x}=0 .
$$

A self-similar solution is as follows:

$$
Z=A \operatorname{erf}(\sqrt{\zeta}), \quad W=\frac{A}{2 t} \operatorname{erf}(\sqrt{\zeta})
$$

The resulting gradual separation of energies according to $(W / Z)^{2} \sim t^{-2}$ is consistent with the numerical result given by Fig. 4. A more detailed analysis would demonstrate that solution (20) could be matched to outer approximations that satisfy the boundary conditions on $|x|=1$. Already the inner development suggests, however, that the self-similar mode weakens the tie between the magnetic and velocity fields and allows the energy separation.

In general, we expect the self-similar mode to emerge only after the large scale oscillatory modes have been viscously dissipated. The emergence of self-similar behavior for resistively dominated plasmas is precluded by the presence of oscillatory decaying eigenmodes for $\eta>\nu$ (see Sec. III D).

\section{Monotonically decaying solutions}

We now consider the case $\eta \simeq \nu$ when viscous and resistive effects act in unison. We use the auxillary functions of Sec. II C, namely

$$
g(x, t)=\frac{1}{2}(Z+W), \quad h(x, t)=\frac{1}{2}(Z-W),
$$

and rewrite Eqs. (7) and (8) in the form

$$
\begin{aligned}
& g_{t}-x g_{x}-\epsilon^{+} g_{x x}=\epsilon^{-} h_{x x}, \\
& h_{t}+x h_{x}-\epsilon^{+} h_{x x}=\epsilon^{-} g_{x x},
\end{aligned}
$$

where

$$
\epsilon^{+}=\frac{\eta+\nu}{2}, \quad \epsilon^{-}=\frac{\eta-\nu}{2} .
$$

Eigenfunction solutions $\sim \exp (\lambda t)$ can be constructed, which decay monotonically for $t \rightarrow \infty$.

In the special case $\eta=\nu$ we have

$$
\begin{aligned}
& \lambda g=x g^{\prime}+\epsilon^{+} g^{\prime \prime}, \\
& \lambda h=-x h^{\prime}+\epsilon^{+} h^{\prime \prime} .
\end{aligned}
$$

Because $\epsilon^{+}$is a small parameter, a boundary layer is present in the region $x \rightarrow 0$. The ideal "outer" solution, given by $g$ $=x^{+\lambda}$ and $h=x^{-\lambda}$, identically satisfies the boundary conditions (9) at $x=1$. To determine $\lambda$, we use the full solution expressed in terms of the confluent hypergeometric (Kummer) functions,

$$
g(x)=g_{0} \frac{x}{\left(\epsilon^{+}\right)^{1 / 2}} M\left(\frac{1}{2}-\frac{\lambda}{2}, \frac{3}{2},-\frac{x^{2}}{2 \epsilon^{+}}\right),
$$




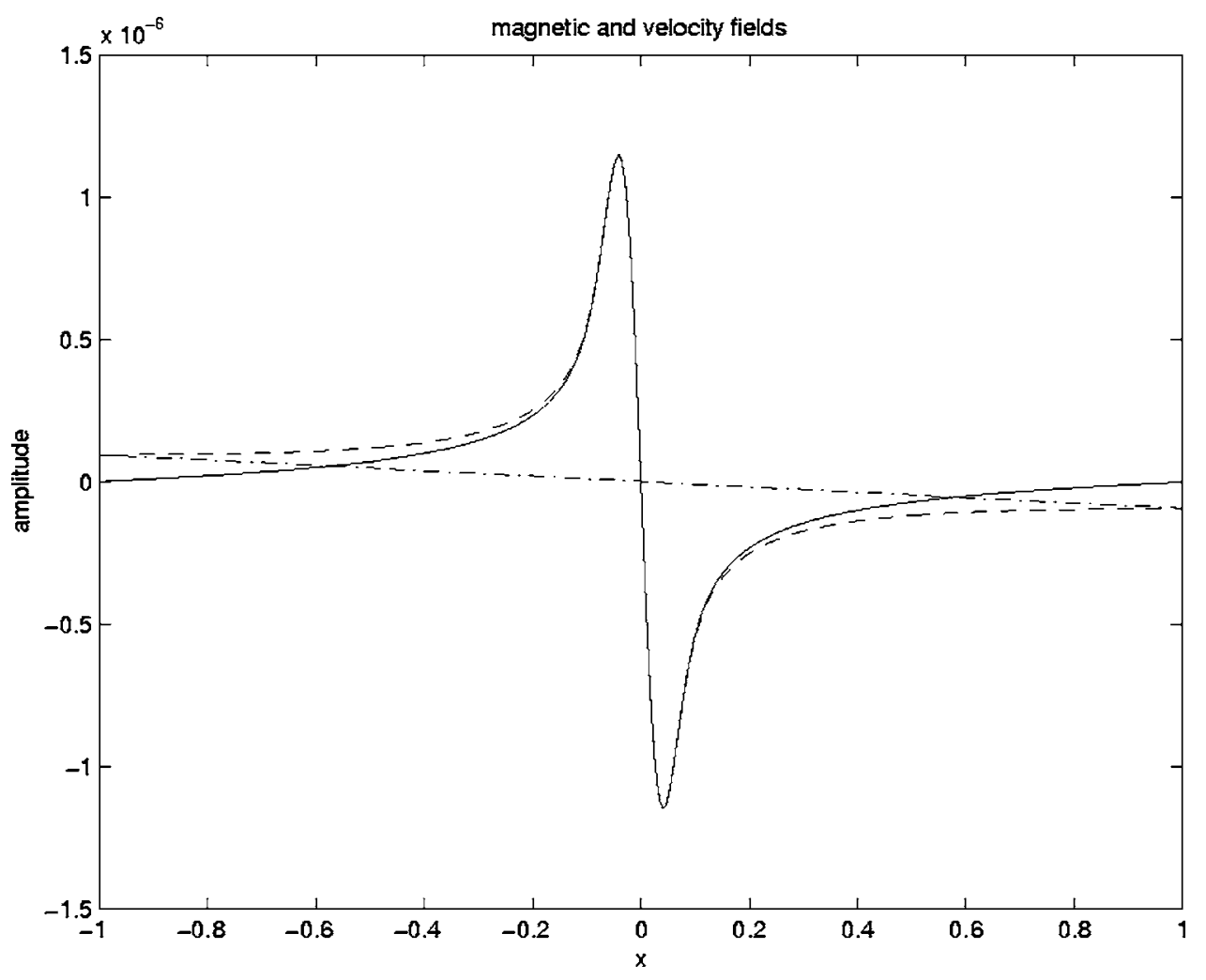

FIG. 5. Fundamental eigenmode for $\eta=10^{-3}, \nu=10^{-3}$. The solid line is $W(x)$, the dashed line is $Z(x)$ and the dotted-dashed line is $Z-W$.

$$
h(x)=h_{0} \frac{x}{\left(\epsilon^{+}\right)^{1 / 2}} M\left(\frac{1}{2}+\frac{\lambda}{2}, \frac{3}{2}, \frac{x^{2}}{2 \epsilon^{+}}\right) .
$$

We are interested in solutions localized around the boundary layer $x=0$. To avoid a blow-up of $h(x)$ as $x /\left(\epsilon^{+}\right)^{1 / 2} \rightarrow \infty$, we must choose $\lambda_{k}=-1-2 k, k=0,1,2, \ldots$, so that the solution is represented as a series in $k$. Clearly the first term in the series is of primary interest, because it describes the asymptotic behavior for $t>1$.

In the case $\lambda=-1$, the general solution simplifies considerably. The function $g(x)$ is expressed in terms of the Dawson integral,

$$
\begin{aligned}
g(x) & =g_{0} \exp \left(-\frac{x^{2}}{2 \epsilon^{+}}\right) \int_{0}^{x} \exp \left(\frac{s^{2}}{2 \epsilon^{+}}\right) \mathrm{d} s \\
& =2^{1 / 2} g_{0} \operatorname{daw}\left(\frac{x}{\left(2 \epsilon^{+}\right)^{1 / 2}}\right),
\end{aligned}
$$

which immediately identifies the scale of the boundary layer $|x| \leqslant\left(\epsilon^{+}\right)^{1 / 2}$. The function $h$ is now linear, and by setting $h_{0}$ $=\epsilon^{+} g_{0}$ we obtain

$$
h(x)=g_{0}\left(\epsilon^{+}\right)^{1 / 2} x .
$$

The fundamental solution is easily extrapolated into the outer field. Specifically, from the asymptotic expansion of the Dawson integral for $x \gg\left(\epsilon^{+}\right)^{1 / 2}$, we have that $g \simeq g_{0}\left(\epsilon^{+}\right)^{1 / 2} / x$, hence $g(x) \simeq h(x)$ and $g^{\prime}(x) \simeq-h^{\prime}(x)$ at the outer boundary $x=1$. It follows that

$$
W(x, t)=\left[g(x)-g_{0}\left(\epsilon^{+}\right)^{1 / 2} x\right] \exp (-t),
$$

$$
Z(x, t)=\left[g(x)+g_{0}\left(\epsilon^{+}\right)^{1 / 2} x\right] \exp (-t),
$$

with $g(x)$ given by Eq. (29), represent the sought-after eigenfunction approximations. These functions, displayed in Fig. 5, satisfy Eq. (9) to an accuracy of order $\epsilon^{+}$and are numerically indistinguishable from eigenmodes computed in the case $\eta=\nu$. Differentiation of the differential equations for $g(x)$ and $h(x)$ shows that higher eigenmodes $g_{k}(x)(k>0)$ are obtained by differentiating $g(x)$, whereas $h_{k}(x)$ are polynomials of order $1+2 k$.

The scaling for the global energy dissipation rate can be deduced by noting that viscous, resistive, and viscoresistive dissipation length scales are comparable when $\eta \simeq \nu$, as are the magnetic and velocity field amplitudes $Z_{0} \simeq W_{0}$. Hence we have

$$
\mathcal{E}_{t} \simeq Z_{0}^{2}\left(\epsilon^{+}\right)^{1 / 2}
$$

where the field amplitude $Z_{0}(t) \simeq Z_{0}(0) \exp (-t)$ in the present case.

We conclude that the most prominent manifestation of the viscoresistive coupling occurs when $\eta$ and $\nu$ have comparable magnitudes. In this case the dissipation length scale $x_{s}=\eta^{1 / 2}=\nu^{1 / 2}$ and monotonic decay rapidly sets in with eigenvalue $\lambda \simeq-1$. More generally, monotonic behavior eventually emerges for $\nu>\eta>0$ (Fig. 3) but only oscillatory modes are present when resistivity is dominant, $\eta>\nu \geqslant 0$ (Fig. 2). To understand why such transition occurs at $\nu \simeq \eta$, we now derive an exact criterion for monotonic decay, as well as an explicit expression for the energy dissipation rate in the case $\nu>\eta$. 


\section{Monotonic decay and energy loss rate}

The previous example indicates that viscosity and resistivity, acting in unison, can lead to exponentially decaying modes in some circumstances. A quantitative condition for the appearance of such modes can be derived by seeking solutions $Z(x, t)=e^{\lambda t} Z(x)$ and $W(x, t)=e^{\lambda t} W(x)$ with a real eigenvalue $\lambda$. If we multiply Eq. (7) by $W^{\prime}(x)$ and Eq. (8) by $Z^{\prime}(x)$, integrate over $(0,1)$, and subtract one equation from the other, we obtain

$$
\lambda Z(1)^{2}=-\eta Z^{\prime}(0)^{2}+\nu\left[W^{\prime}(0)^{2}-W^{\prime}(1)^{2}\right] .
$$

This equation precisely describes the monotonic decay in the late phase of Fig. 3. The fact that the magnetic and velocity fields do not appear symmetrically in this condition is a reflection of the boundary conditions imposed in Eq. (9).

If we assume that $\lambda<0$ and $\left|W^{\prime}(1)\right| \ll\left|W^{\prime}(0)\right|$, as required for consistency with the numerical solutions, we obtain a simple necessary condition for monotonic decay,

$$
Z^{\prime}(0)>\left(\frac{\nu}{\eta}\right)^{1 / 2} W^{\prime}(0) \text {. }
$$

For $\nu \simeq \eta$, the magnetic field gradient at the origin must exceed the velocity gradient, and this is indeed the case for the particular solution derived for $\eta=\nu$.

Suppose now that $\nu>\eta$. Equation (35) shows that monotonicity requires a strong separation between the magnetic and velocity field amplitudes. Indeed, the scalings $W$ $\simeq W^{\prime}(0) x_{s}$ and $Z \simeq Z^{\prime}(0) x_{s}$ suggest that monotonic decay can set in when the magnetic energy is larger than the kinetic energy by a factor of order $\nu / \eta$, which is consistent with the preferential dissipation of kinetic energy by viscosity (see also the Appendix). On the other hand, in the case $\eta>\nu$, magnetic energy is dissipated more efficiently, and Eq. (35) is impossible to satisfy. In this case oscillatory decay should result, in agreement with the numerical solution. Figure 2 shows an example of oscillatory eigenfunctions, associated with energy equipartition.

We can also employ Eq. (34) to estimate the rate of decay $\lambda$. A simple upper limit can be obtained by neglecting the weak velocity contribution and assuming that the viscoresistive scale determines the field gradient. Assuming $Z(1) \simeq 1$ and $Z^{\prime}(0) \simeq x_{s}^{-1}$, we obtain

$$
\lambda \simeq-\sqrt{\eta / \nu} .
$$

A key point is that, for fixed $\eta<\nu$, the decay rate diminishes with increasing $\nu$. A related result is that the kinetic energy is a small fraction of order $\lambda^{2}$ of the magnetic energy. Similar considerations for the global resistive energy loss rate lead to

$$
\mathcal{E}_{t} \simeq \eta \int_{0}^{1}\left(Z_{x}\right)^{2} \mathrm{~d} x \simeq Z_{0}^{2} \eta^{1 / 2}\left(\frac{\eta}{\nu}\right)^{1 / 4}
$$

which generalizes the result of Sec. III C.

\section{E. Summary}

We have considered the viscous and resistive damping of perpendicularly polarized shear waves near a planar magnetic $X$-point. A surprising result is the sensitivity of the de- cay profile and the global energy loss rate to the relative magnitudes of the viscous and resistive dissipation coefficients.

When either viscosity or resistivity vanishes, the solution is characterized by oscillatory decaying eigenmodes that maintain equipartition between the magnetic and kinetic energies (see Sec. III A). This behavior persists in plasmas dominated by resistivity, $\eta>\nu>0$. The situation changes markedly when viscosity is the dominant dissipation mechanism, $\nu>\eta>0$. In the transitional case $\eta \simeq \nu$ the energy decays exponentially, with the fundamental eigenvalue $\lambda=-1$. More generally, the damping is characterized by initial oscillations that gradually give way to exponential decay at sufficiently long times. The bulk of the wave energy is rapidly removed during the oscillatory stages on the viscous time scale $\nu^{-1 / 2}$ (assuming $\nu \gg \eta$ ), but the later monotonic decay is associated with a marked separation in the global magnetic and kinetic energies. This stage corresponds to the emergence of a viscoresistive scale $x_{s}=(\eta \nu)^{1 / 4}$ that provides asymptotic exponential decay in which the magnetic energy is dominant. Preferential damping of the kinetic energy component can also occur due to the emergence of a self-similar mode for large times in the limit $\eta \rightarrow 0$. This mode, however, is energetically insignificant.

\section{STEADY SOLUTIONS AND ANISOTROPIC VISCOSITY}

So far we used the MHD equations in order to describe the dynamics of transient shear waves near a planar magnetic $X$-point. It is also possible to obtain exact steady solutions describing the axial magnetic field and velocity disturbances. The relative simplicity of the steady-state analysis makes it possible to discuss the effects of anisotropic viscous transport in magnetized plasmas.

\section{A. Viscosity in a magnetized plasma}

Our work is partly motivated by the possibility that viscosity can lead to significant observational effects in solar active regions. Of particular interest are recent measurements of relatively slow speeds of reconnection jets in solar flares. ${ }^{14-16}$ The observations appear to be inconsistent with standard nonviscous reconnection models that predict the reconnection outflow speed to be determined by the Alfvén speed. Because viscosity results in slower reconnection outflows, ${ }^{2}$ it can reconcile theoretical models with solar observations. Although viscous effects are typically modeled by an isotropic diffusion term with a single scalar viscosity coefficient $\nu,{ }^{7,9,17}$ one should remember that viscous stresses are anisotropic in magnetized plasmas. ${ }^{18-20}$ Theoretical modeling that incorporates the full anisotropic viscous stress tensor for an arbitrary magnetic field strength is beyond the scope of this paper. Below we illustrate the effects of anisotropic viscous transport by presenting a comparison of steady solutions valid for weak and strong magnetic fields.

In sharp contrast to the resistivity tensor, the viscous stress tensor $\sigma_{i j}$ is strongly modified in the presence of a magnetic field $\mathbf{B}$. The only component of $\sigma_{i j}$ that remains unaffected is the component corresponding to the momentum 
flux along $\mathbf{B}$, which is driven by the velocity gradient along B. The magnetic field alters the form of the viscosity when the proton mean free path exceeds the gyroradius, $\omega_{p} \tau_{p} \gg 1$, where $\omega_{p}=e B /\left(m_{p} c\right)$ is the proton cyclotron frequency and $\tau_{p} \simeq 0.75 T^{3 / 2} / n$ is the mean time (in seconds) between momentum-changing collisions. ${ }^{18}$

As an illustrative example, assume the following reference values that may characterize an active region in the solar corona: length scale $L=10^{9.5} \mathrm{~cm}$, number density $n$ $=10^{9} \mathrm{~cm}^{-3}$, magnetic field $B=10^{2} \mathrm{G}$, proton temperature $T$ $=10^{6} \mathrm{~K}$. The corresponding Alfvén speed is $v_{A}=10^{9} \mathrm{~cm} \mathrm{~s}^{-1}$. The dimensionless resistivity, based on the classical collisional value $\eta \sim T^{-3 / 2}$ is of order $\eta=10^{-14.5}$, whereas the dimensionless viscosity $\nu \sim T^{5 / 2}$ is of order $\nu=10^{-4.5}$. It follows that $\nu \gg \eta$ so that viscosity can strongly influence the coronal dynamics. At the same time, $\omega_{p} \tau_{p} \simeq 10^{6} \gg 1$, and hence the viscous transport is highly anisotropic in the solar corona. This is why the quantitative expressions derived in this paper can be applied only in weak field regions (around magnetic nulls) of the solar plasma. We believe, however, that the qualitative behavior of our solutions can guide more detailed studies.

\section{B. Steady-state solution with isotropic viscosity}

Suppose the magnetic field is weak enough for the viscous term to be described by the Laplacian form. Returning to the MHD equations of Sec. II A, consider a steady merging solution for the axial components of velocity $W(x)$ and magnetic field $Z(x)$. Setting $\partial_{t}=0$ in Eqs. (7) and (8) gives

$$
Z^{\prime}=\eta \nu \frac{1}{x}\left(\frac{1}{x} Z^{\prime \prime}\right)^{\prime}
$$

Advection and dissipation must be balanced in steady state. Hence the viscoresistive scale $x_{s}=(\eta \nu)^{1 / 4}$ is the only spatial scale of interest. Note for clarity that steady merging is driven by forces at the external boundaries. Hence the sought-after solution does not have to satisfy the boundary conditions given by Eq. (9).

The solution is selected by assuming that the field gradient is localized around $x=0$. The odd profile of the axial magnetic field takes the form of the error function with an amplitude $Z_{0}$,

$$
Z(x)=Z_{0} \operatorname{erf}\left(\frac{x}{\sqrt{2} x_{s}}\right)
$$

The corresponding velocity is proportional to the axial magnetic field,

$$
W(x)=\left(\frac{\eta}{\nu}\right)^{1 / 2} Z(x) .
$$

This result reflects a balance between the viscous and magnetic forces in steady solutions. The flow amplitude $W_{0}$ must scale as $(\eta / \nu)^{1 / 2} Z_{0}$. Finally, arbitrary constant values $Z(0)$ and $W(0)$ can be added to the steady solutions.

Evaluating the global dissipation rate using the length scale $x_{s}=(\eta \nu)^{1 / 4}$ gives

$$
\nu \int_{0}^{1}\left(W_{x}\right)^{2} \mathrm{~d} x \simeq \eta \int_{0}^{1}\left(Z_{x}\right)^{2} \mathrm{~d} x \simeq Z_{0}^{2} \eta^{1 / 2}\left(\frac{\eta}{\nu}\right)^{1 / 4} .
$$

Both viscous and resistive dissipation rates decrease as viscosity increases. We conclude that the emergence of the viscoresitive scale $x_{s}$ leads not only to the separation of the magnetic field and velocity amplitudes for $\nu>\eta$ but also to the decline in the global energy dissipation rate. This is fully consistent with the dissipation rate of Eq. (37), obtained for the transient solution.

\section{Steady-state solution with anisotropic viscosity}

Now consider the opposite limit of a strong magnetic field that alters the viscous stress tensor significantly. When $\omega_{p} \tau_{p} \gg 1$, the dominant terms in the Braginskii viscous stress tensor are given by

$$
\sigma_{i j}=\nu\left(3 \frac{B_{i} B_{j}}{B^{2}}-\delta_{i j}\right)\left(\frac{B_{k} B_{l}}{B^{2}} \partial_{l} v_{k}-\frac{1}{3} \nabla \cdot \mathbf{v}\right),
$$

where all quantities are dimensionless. ${ }^{18,19}$ We assume incompressibility and consider steady solutions with a dominant axial magnetic field, $B \simeq Z(x)$, and $W=W(x)$ as before. Keeping only the leading-order terms with respect to $B^{-1}$, we obtain the following approximate expression for the viscous force:

$\partial_{j} \sigma_{i j}=-\nu \nabla\left(\frac{B_{x}}{Z} W^{\prime}\right)+3 \nu\left[\partial_{x}\left(\frac{B_{x}^{2}}{Z^{2}} W^{\prime}\right)+\partial_{y}\left(\frac{B_{x} B_{y}}{Z^{2}} W^{\prime}\right)\right] \hat{\mathbf{z}}$.

Replacing the Laplacian term by this form of the viscous force in Eqs. (7) and (8), we obtain

$$
\begin{aligned}
& Z^{\prime}+3 \nu\left(Z^{-2} x W^{\prime}\right)^{\prime}=0, \\
& x W^{\prime}+\eta Z^{\prime \prime}=0 .
\end{aligned}
$$

The resulting equation for the axial magnetic field,

$$
Z^{\prime}=3 \eta \nu\left(Z^{-2} Z^{\prime \prime}\right)^{\prime},
$$

should be contrasted with Eq. (38) that was based on the isotropic viscosity.

Equation (46) can be integrated twice to yield

$$
\left(Z^{\prime}\right)^{2}=\frac{1}{6 \eta \nu} Z^{4}+a Z^{3}+\left[Z^{\prime}(0)\right]^{2},
$$

where $a$ is an arbitrary constant. The solution for $Z(x)$ can be formally expressed in terms of Jacobi elliptic functions. For comparison with the solution for isotropic viscosity, we initially assume $Z(x)$ to be an analytic odd function, which implies $a=0$. In addition if the magnitude of $Z(1)$ is large enough, we can ignore the term $Z^{\prime}(0)$ far from $x=0$ and obtain

$$
Z(x \rightarrow 1) \simeq \frac{(6 \eta \nu)^{1 / 2} Z(1)}{(6 \eta \nu)^{1 / 2}+(1-x) Z(1)},
$$

and the corresponding expression for $x \rightarrow-1$. The characteristic length scale in the strong-field limit is given by $(\eta \nu)^{1 / 2}$ rather than $(\eta \nu)^{1 / 4}$. Similarly the ratio $Z_{0} / W_{0}$ of typical mag- 


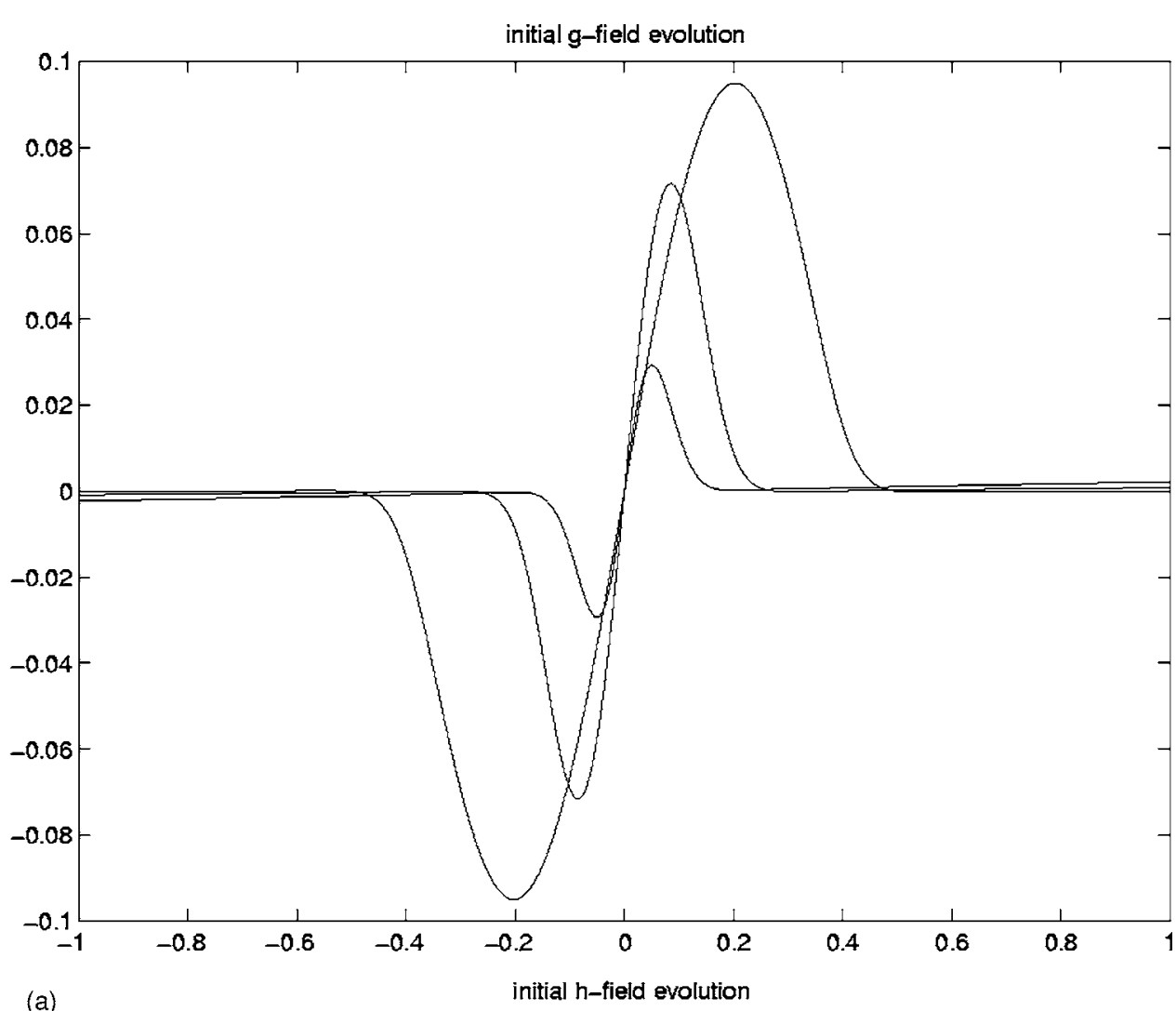

(a)

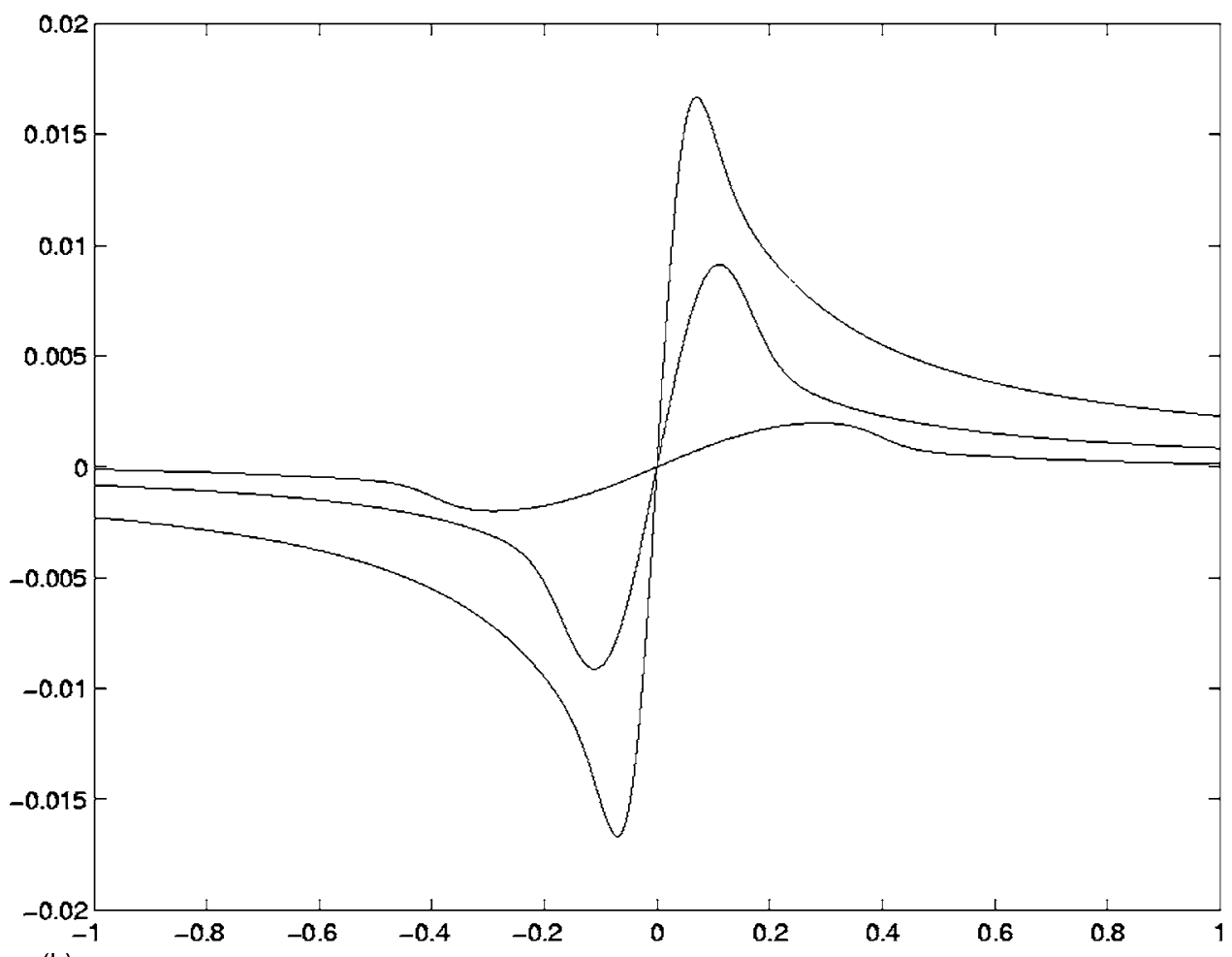

FIG. 6. Time slices showing initial wave decay for $\eta=10^{-3}, \nu=10^{-2.5}$. The decline of $g$ in (a) contrasts with the growth of $h$ in (b). Both plots show wave profiles at $t=0.75,1.35,2.25$ Alfvén times, with peaks moving systematically inwards.

(b)

nitudes of the magnetic field and velocity scales as $\nu / \eta$ rather than $(\nu / \eta)^{1 / 2}$ in the weak-field limit.

The most notable qualitative difference of the field profile $Z(x)$ in the strong-field limit, compared with the errorfunction profile in the weak-field limit, is a localized gradient of the magnetic field at the outer boundaries $x= \pm 1$. It should be remembered, of course, that $Z(1)$ needs to be large enough to justify the approximate form of the anisotropic viscous stress tensor. Probably more critical is the breakdown of the approximation at $x=0$, where $Z \rightarrow 0$. This could lead to another current layer at $x=0$, which would be analogous to the weak-field case. A similar solution can be derived 
in the case of a large constant background field $Z(0) \neq 0$. The field profile is then $Z(x)-Z(0) \sim \sinh \left[x /(3 \eta \nu)^{1 / 2} Z(0)\right]$, which is also localized at the outer boundary. By contrast, when $Z(0) \neq 0$, the formal weak-field solution remains localized at the origin, since its error-function profile is simply shifted by $Z(0)$.

The steady solutions described above confirm the nontrivial relationship between the magnetic field strength and the viscous plasma dynamics. We should bear in mind, however, that by choosing $a \neq 0$ in Eq. (47) we can construct nonanalytic solutions for $Z(x)$. In particular, taking $a$ $\sim \operatorname{sgn}(x)$ results in an odd solution for $Z(x)$ with $Z(0)=0$ and with the electric current localized at $x=0$ on the scale $(\eta \nu)^{1 / 2}$. This behavior is similar to that of the weak-field solution, but the price paid for the current localization is a weak discontinuity of $Z(x)$ at the origin. Formally the discontinuity implies the presence of a delta-functional term in Eq. (46). A similar situation has already been described for two-dimensional viscoresistive reconnection. ${ }^{8}$ The discontinuous solution may have physical validity if the higherorder terms, neglected in Eq. (46), lead to a smooth solution at the origin.

These arguments emphasize the importance of studying the dynamical accessibility of the steady-state solutions, which would allow us to pick out the correct steady solution by following the temporal evolution of the system. The analysis of the dynamical accessibility of steady-state models will be pursued elsewhere, but it seems apt to mention that our preliminary dynamic computations indicate the development of odd solutions localized to the origin both for the isotropic viscosity and for anisotropic viscosities that approximate the full viscous stress tensor. Notably, the steady solutions, based on the isotropic viscosity, yield dissipation scalings that are consistent with transient models of Sec. III.

\section{DISCUSSION}

We have discussed the viscoresistive damping of perpendicularly polarized shear waves at a planar magnetic $X$-point. By using a combination of numerical and analytical techniques, we have shown that the form of the energy decay depends strongly on the relative magnitudes of the viscous and resistive dissipation coefficients. Three distinct length scales are present in the problem, which can be ordered according to $\eta^{1 / 2}<(\eta \nu)^{1 / 4}<\nu^{1 / 2}$ in the case of a viscously dominated plasma. The relevant length scale defines the gradients of the velocity and magnetic fields and hence the rate of energy dissipation in the system.

In general, the initial decay phase is dominated by oscillatory eigenmodes that maintain equipartition between the magnetic and kinetic energies. Oscillatory modes, acting on the scale $\eta^{1 / 2}$, also control the asymptotic decay when resistivity is dominant, $\eta>\nu$. Energy equipartition is found to break down only for $\nu>\eta$. In this case the oscillatory modes eventually give way to exponential decay, and dissipation occurs on the viscoresistive scale $x_{s}=(\eta \nu)^{1 / 4}$. When $\nu>\eta$ $>0$, the kinetic energy initially dissipates according to $\mathcal{E}_{t}$ $\sim \nu^{1 / 2}$ [see Eq. (18)]. Later the decay is determined by the slow dissipation of the magnetic energy, which scales as $\mathcal{E}_{t}$ $\sim \eta^{1 / 2}(\eta / \nu)^{1 / 4}$ [see Eq. (37)]. An identical dissipation scaling holds for the steady solution, driven by a shear flow. We emphasize that, although the asymptotic dissipation rate is slow for $\eta \ll \nu \ll 1$, the early oscillatory phases of viscous damping are capable of releasing a large fraction of the wave energy quite efficiently, on a scale of a few hundred Alfvén times. ${ }^{9,21}$ This may have interesting implications for the processes of energy release in magnetized plasmas.

The principal role of viscosity was noted some time ago in the context of the damping of Alfvén waves in the solar corona. When the waves propagate in a region with a large gradient of the Alfvén speed, they experience phase mixing that enhances the wave damping rate. For typical parameters of the solar corona, the damping length has been found to be determined by viscous rather than resistive dissipation, and the damping rate has been found to scale as $\nu^{1 / 3} \cdot{ }^{17}$ This scaling is different from the viscous dissipation rate $\mathcal{E}_{t} \sim \nu^{1 / 2}$ derived here because phase mixing in a quasi-onedimensional background magnetic field produces "corrugated" dissipation layers as opposed to a single dissipating sheet. Aside from these differences, which can be traced to variations in the field geometry, the decay phenemena witnessed in the more recent phase mixing studies, as well as the techniques used to model them, ${ }^{21}$ are well represented in the present calculations.

We conclude by stressing that $\nu \gg \eta$ is expected in many astrophysical and laboratory applications. More work is needed to understand viscous magnetic merging and reconnection under realistic astrophysical conditions. A prime example is the solar corona, where a key observational clue to the role of viscosity may be provided by the relatively slow speed of reconnection outflow jets in solar flares. ${ }^{16}$ Interesting effects are expected because the background magnetic field should strongly modify the viscous transport. Then the Braginskii viscous stress tensor must be used to obtain a more accurate description of the plasma dynamics. Although few analytical solutions of this sort have been derived so far, ${ }^{4,19}$ our present study suggests that the global energy losses should be strongly dependent on the geometry of the magnetic field.

\section{ACKNOWLEDGMENTS}

This work was supported by Marsden (02-UOW-050 MIS), by NSF (Grant No. ATM-0136718), and by NASA (Grants Nos. NAG5-11797 and NNG05GM43G).

\section{APPENDIX: TRAVELING WAVE SOLUTIONS FOR SMALL TIMES}

To show the generality of the derived dissipation scalings, we now use the formalism of Sec. III C to describe the damping of propagating waves. The preferential damping of the kinetic energy occurs in the case $\nu>\eta$. We assume that $h=0$ initially and consider the mode

$$
g(x, t)=G(t) e^{i k(t) x} .
$$

Equation (22) shows that 


$$
\dot{k}=k, \quad \dot{G}=-\epsilon^{+} k^{2} G .
$$

Obviously, the damping will be strongest when the wave number $k(t)$ has increased to the level where $\left|g_{x}\right|=|G(t) k(t)|$ is steepest. For a global initial disturbance with $k(0) \simeq 1$ this occurs at the time

$$
t=t_{m} \simeq \frac{1}{2} \ln \left(\frac{1}{\epsilon^{+}}\right),
$$

and so $k\left(t_{m}\right)=\left(\epsilon^{+}\right)^{-1 / 2}$. Since the damping layer now has the thickness $x \simeq k^{-1}=\left(\epsilon^{+}\right)^{1 / 2}$, the maximum dissipation rate scales as

$$
\mathcal{E}_{t}\left(t_{m}\right) \simeq G_{0}\left(\epsilon^{+}\right)^{1 / 2},
$$

where $G_{0}=G(0)$ defines the initial wave amplitude.

These expressions, although exact only in the special case $\eta=\nu$, should provide a reasonable approximation in the early phases of the damping even for $\epsilon^{-} \neq 0$. This follows from the observation that $h \simeq 0$ must hold before significant dissipation sets in $\left(t \ll t_{m}\right)$ due to the ideal constraint of energy equipartition. Growth, however, will eventually be induced according to Eq. (23), $h_{t} \simeq \epsilon^{-} g^{\prime \prime}$. Taking $h$ $\simeq H(t) \exp [i k(t) x]$ with $H$ small implies that

$$
H(t)=\frac{\epsilon^{-}}{\epsilon^{+}}\left[G(t)-G_{0}\right]
$$

for $t \ll t_{m}$. The important point is that the growth in $h$ drives a weak asymmetry in the amplitudes of the $W$ and $Z$ fields.

Suppose $\nu>\eta$ so that $\epsilon^{-}<0$. Then $H$ will have the same sign as $G$ [using (A5)] and the magnitude of $Z=g+h$ will dominate $W=g-h$. Such behavior is confirmed in Fig. 6 which contrasts the decline of the $g$-field [Fig. 6(a)] with the growth of the induced component $h$ [Fig. 6(b)] for the initial phase $t<t_{m}$. The plots assume initial conditions of the form $g(x, 0)=\sin (\pi x), h(x, 0)=0$. The solution quantifies the traveling wave dissipation in the case $\nu>\eta$ when the damping is predominantly viscous. A similar argument shows that the kinetic energy will be dominant when the damping is predominantly resistive.

${ }^{1}$ E. N. Parker, Spontaneous Current Sheets in Magnetic Fields (Oxford University Press, Oxford, 1994)

${ }^{2}$ W. Park, D. A. Monticello, and R. B. White, Phys. Fluids 27, 137 (1984).

${ }^{3}$ R. B. Fabling and I. J. D. Craig, Phys. Plasmas 3, 2243 (1996).

${ }^{4}$ Y. E. Litvinenko, Sol. Phys. 229, 203 (2005).

${ }^{5}$ J. V. Hollweg, J. Geophys. Res., [Atmos.] 90, 7620 (1985).

${ }^{6}$ S. V. Bulanov, S. G. Shasharina, and F. Pegoraro, Plasma Phys. Controlled Fusion 32, 377 (1990).

${ }^{7}$ A. B. Hassam and R. P. Lambert, Astrophys. J. 472, 832 (1996).

${ }^{8}$ V. S. Titov and E. R. Priest, J. Fluid Mech. 348, 327 (1997).

${ }^{9}$ I. J. D. Craig, Y. E. Litvinenko, and T. Senanayake, Astron. Astrophys. 433, 1139 (2005)

${ }^{10}$ I. J. D. Craig and A. N. McClymont, Astrophys. J. 481, 996 (1997).

${ }^{11}$ I. J. D. Craig and S. M. Henton, Astrophys. J. 450, 280 (1995).

${ }^{12}$ P. G. Watson and I. J. D. Craig, Astrophys. J. 505, 363 (1998).

${ }^{13}$ Y. E. Litvinenko and I. J. D. Craig, Sol. Phys. 189, 315 (1999).

${ }^{14}$ D. E. McKenzie, Sol. Phys. 195, 381 (2000).

${ }^{15}$ D. E. McKenzie and H. S. Hudson, Earth, Planets Space 53, 577 (2001).

${ }^{16}$ A. Asai, T. Yokoyama, M. Shimojo, and K. Shibata, Astrophys. J. 605, L77 (2004).

${ }^{17}$ J. Heyvaerts and E. R. Priest, Astron. Astrophys. 117, 220 (1983).

${ }^{18}$ S. I. Braginskii, Rev. Plasma Phys. 1, 205 (1965).

${ }^{19}$ J. V. Hollweg, Astrophys. J. 306, 730 (1986).

${ }^{20}$ D. Montgomery, J. Geophys. Res., [Atmos.] 97, 4309 (1992).

${ }^{21}$ I. J. D. Craig and G. Fruit, Astron. Astrophys. 440, 375 (2005). 